\title{
Ensino e Cuidado em Saúde LGBTI+: Reflexões no Contexto da Pandemia da Covid-19
}

\author{
LGBTI + Teaching and Health Care: Reflections in the Context of the Covid-19 Pandemic
}

\author{
Ademir Lopes Junior' (D), Gustavo Antonio Raimondi" (D), Daniela Murta"l' (1D) \\ Tchayra Tatiane Souzalv (i), Rita Helena Borret ${ }^{I I,,}$ (iD)
}

\begin{abstract}
RESUMO
Introdução: As Diretrizes Curriculares Nacionais para os cursos de Graduação em Medicina (DCN) de 2014 definem que o(a) graduando(a) deve ser formado(a) para abordar a diversidade biológica, étnico-racial, de gênero e orientação sexual. Entretanto, essa temática costuma ser invisibilizada, reiterando a LGBTI+fobia institucional que perpetua desigualdades e iniquidades no ensino e cuidado em saúde. Essa situação se tornou mais explícita durante a pandemia da Covid-19.

Objetivo: Analisar as DCN sob a ótica das diversidades de gênero e sexual, de modo a problematizar "o que" e "como" pode ser aprimorado na educação médica em relação às questões LGBTI+.

Desenvolvimento: Considerando que a aprendizagem de adultos ocorre a partir da problematização de vivências do cotidiano, serão apresentados três casos relativos ao atual momento da pandemia da Covid-19, com base nos quais se podem abordar temas sobre a saúde LGBTI+ no curso médico. Após, serão problematizadas algumas competências específicas sobre saúde LGBTI+ que podem ser aprimoradas durante a formação médica, inclusive no período da pandemia da Covid-19, a fim de visibilizar a temática LGBTI+ no currículo como uma estratégia de combater a LGBTI+fobia na escola médica e promover o cuidado integral em saúde.
\end{abstract}

Conclusão: É possível abordar sobre a saúde LGBTI+ a partir de situações vivenciadas durante a pandemia da Covid-19. Entretanto, é necessário ter clareza de como as DCN se traduzem em competências específicas sobre saúde LGBTI+. Essa pode ser uma das estratégias a fim de tornar os currículos mais acolhedores e compromissados com as necessidades de saúde dessa população.

Palavras-chave: Minorias Sexuais e de Gênero; Preconceito; Pandemia; Educação Médica.

\section{ABSTRACT}

Introduction: The 2014 National Curriculum Guidelines ("DCNs") determine that undergraduates should be trained to address biological, ethnicracial, gender and sexual orientation diversity. However, academic medical training only occasionally addresses this theme, which perpetuates inequality and prevents physicians from being adequately trained to serve this population. The reorganization of curricular activities due to the Covid-19 pandemic may have aggravated this situation and this invisibility emphasizes institutional LGBTI+phobia.

Objective: To shed light on the LGBTI+ theme in academic medical training is one of the strategies to tackle LGBTI+phobia in medical school. This essay will analyze the "DCNs" from the perspective of gender and sexual diversity, recommending related competencies on LGBTI+ health that should feature in the course, including the Covid-19 pandemic period.

Development: We will present three cases related to the current moment of the Covid-19 pandemic, from which topics on LGBTI + health could be discussed in academic medical training. After analyzing the DCNs and the needs arising from these cases, some competencies in LGBTI+ health will be proposed for undergraduate medicine students; considering that adult learning results from critically questioning everyday experiences.

Conclusion: we believe that it is possible to approach LGBTI+ health from situations experienced during the Covid-19 pandemic. However, it is necessary to be clear how the DCNs translate to LGBTI+ health-related skills. This might be one of the strategies to make academic medical training more welcoming and committed to the health needs of this group.

Keywords: Sexual and Gender Minorities; Prejudice; Pandemic; Medical Education.

'Universidade de São Paulo, São Paulo, São Paulo, Brasil.

"Universidade Federal de Uberlândia, Uberlândia, Minas Gerais, Brasil.

"'Universidade Estácio de Sá, Rio de Janeiro, Rio de Janeiro, Brasil.

"Vaculdade da Saúde e da Ecologia Humana, Vespasiano, Minas Gerais, Brasil.

${ }^{\vee}$ Escola Nacional de Saúde Pública, Rio de Janeiro, Rio de Janeiro, Brasil.

Correspondência

Ademir Lopes Junior.

Rua Minerva, 149, ap. 92, Perdizes, São Paulo, SP, Brasil. CEP: 05007-030.

E-mail: ad.lopesjunior@gmail.com

Recebido em 11/08/20; Aceito em 07/09/20. 


\section{INTRODUÇÃO}

As Diretrizes Curriculares Nacionais para os cursos de Graduação em Medicina (DCN) mencionam, em seu artigo $5^{\circ}$, que o(a) graduando(a) deve ser formado(a) para abordar a diversidade biológica, étnico-racial, de gênero e orientação sexual'. Entretanto, se, em condições normais, as pessoas lésbicas, gays, bissexuais, travestis, transexuais, intersexos e demais expressões de identidade de gênero e orientação sexual (LGBTI+) já são excluídas e vulnerabilizadas pela LGBTI+fobia, no cenário da pandemia da coronavirus disease (Covid-19), isso se agravou². Desrespeito ao nome social e medo de preconceito são algumas das facetas dessa violência que afastam essas pessoas dos serviços de saúde. De forma semelhante, há que se ter atenção para que a temática da saúde LGBTI+ não se torne ainda mais invisível nos cursos médicos a partir da reorganização das atividades curriculares em decorrência da pandemia, como a transferência de aulas presenciais para o ambiente virtual por conta do distanciamento social e o enfoque dos cenários de prática no atendimento a pessoas com problemas respiratórios.

Considerando que a atual situação de pandemia da Covid-19 explicitou a importância de termos um olhar e um cuidado atentos para as populações vulnerabilizadas, como a LGBTI+, o presente ensaio analisará as $\mathrm{DCN}^{1}$ sob a ótica da diversidade de gênero e sexual. Para isso, serão apresentadas três cenas com pessoas LGBTI+, construídas a partir das experiências de ensino e de cuidado dos(as) autores(as) em cenários reais durante a pandemia de Covid-19. Optou-se pela metodologia de apresentação de casos a fim de expor possibilidades de como podem surgir essas temáticas no contexto atípico de uma pandemia. Afinal, o primeiro passo para discussão é percebê-lo e identificá-lo no cotidiano. Portanto, os casos visam explicitar de que forma - "o que" e "como" - aparecem essas questões na prática médica. A partir disso, serão propostas competências profissionais que poderiam ser trabalhadas e que foram elaboradas a partir da intersecção das DCN com os debates contemporâneos sobre diversidade sexual e de gênero.

\section{“ENTRE TAPAS E BEIJOS(?)": AS VIOLÊNCIAS E AS (IN)VISIBILIDADES CONTRA A POPULAÇÃO LGBTI+}

- Cena 1: Jonas, 33 anos, homem cis preto, gay, queixa-se de falta de ar há três dias. Acha que é Covid-19. Exame físico sem alterações. O médico levanta a hipótese de episódio de ansiedade. Ao ser perguntado, Jonas diz que a vida não está fácil, trabalhava como motorista de aplicativo, e, após a pandemia, sua renda diminuiu muito. O médico orienta que Jonas tire alguns dias de "férias" para descansar e se sentir melhor. Entretanto, Jonas não conseguiu contar que tem tido brigas recorrentes com seu atual namorado que é muito ciumento. É a primeira vez que namora um homem branco, Fábio, 34 anos, economista que trabalha em uma grande rede bancária. Sente que Fábio o humilha algumas vezes porque ganha mais e só o procura para relações sexuais.

\section{Visibilizar a pessoa LGBTI+ e praticar o cuidado centrado} na pessoa de forma afirmativa

Como cuidar se não se conhece o outro, suas preocupações e seu contexto? Não seria possível identificar conflitos conjugais nesse caso se o relacionamento afetivo de Jonas fosse invisibilizado. Só seria possível abordá-lo se o paciente pudesse expressá-lo por meio de uma escuta atenta, de uma comunicação clínica afirmativa e de um cuidado centrado na pessoa ${ }^{3}$. Assim, a primeira etapa para cuidar de uma pessoa $\mathrm{LGBTI+,} \mathrm{como} \mathrm{no} \mathrm{caso} \mathrm{descrito,} \mathrm{é} \mathrm{considerar} \mathrm{que} \mathrm{alguém}$ pode não ser cis-heterossexual, reduzindo as pressuposições cis-heteronormativas que perpetuam a LGBTI+fobia ${ }^{4}$.

São comuns relatos de atendimentos em saúde, bem como os processos de ensino-aprendizagem, que descrevem exames físicos inadequados, de coleta de informações inapropriadas, de experiências de cuidado violentas que tornam o serviço pouco acolhedor para pessoas LGBTI+5. Por isso, é preciso compreender que, por exemplo, algumas pessoas trans podem ter desconforto com os seus genitais e suas mamas, e que lésbicas e mulheres bissexuais que mantêm relações sexuais penetrativas devem realizar a colpocitologia oncótica, quando na faixa etária de rastreamento. Explicitando, assim, que as condutas clínicas relacionadas à saúde LGBTI+ deveriam ser sempre baseadas em evidências científicas, como demonstra o artigo $12^{\circ}$ das $\mathrm{DCN}^{3}$, e não em "achismos" ou valores morais (geralmente excludentes).

A semiologia, outro exemplo, em vez de perguntar a um homem "Você já teve relação sexual com uma mulher?", pode ensinar "Você já teve relação sexual?"; se sim, "Com alguma mulher, homem ou ambos?". Não se deve supor que toda pessoa com expressão masculina seja um homem cisgênero; afinal, pode ser um homen trans. $O$ ensino das habilidades de comunicação deve incluir perguntas que abranjam a diversidade sobre identidade de gênero ("Como você gostaria de ser chamado(a)?" e "Como você se identifica em relação ao gênero?"), práticas sexuais ("Você gostaria de relatar suas práticas sexuais?", "Você tem relação anal?" e "Você penetra ou é penetrado(a)?”) e o reconhecimento de sentimentos e expressões não verbais que indiquem julgamento e não aceitação da diversidade ${ }^{6}$.

\section{Conhecer conceitos de interseccionalidades e vulnerabilidades LGBTI+}

Jonas não é apenas gay. É gay preto, motoboy, que namora outro gay branco rico. As diversidades biológica, 
étnico-racial, de gênero ou orientação sexual recebem valores e simbologias distintos em cada contexto social. Nogueira7 aponta que a população negra brasileira é formada e compreendida a partir de um processo sócio-histórico de escravização por mais de 300 anos, da prática de estupros de mulheres negras que vão dar origem ao romantizado processo de miscigenação do povo e de um processo de abolição da escravatura tardio e que não se deu com políticas públicas de inclusão, reparação ou garantia do reconhecimento de cidadania. Hoje, $55,8 \%$ da população que se reconhece e autodeclara como parda ou preta vivencia o racismo como parte do seu processo de saúde e adoecimento ${ }^{8}$.

Reconhecer, assim, as interseccionalidades ${ }^{9}$ implica compreender como as relações socioculturais interagem com aspectos individuais e coletivos na construção de condições de fragilidade e perigo. Várias políticas públicas (Política Nacional de Atenção Integral à Saúde da Mulher ${ }^{10}$, Política Nacional de Atenção Integral à Saúde da População Negra"1 e Política Nacional de Saúde Integral de Lésbicas, Gays, Bissexuais, Travestis e Transexuais ${ }^{12}$ ) reconhecem as lacunas sobre esses temas no currículo de graduação. Jonas é um homem cis, negro e gay, e, a partir dessas interseccionalidades e dos marcadores sociais da diferença, sua experiência de viver e seus processos de saúde e adoecimento diferem daqueles vividos por outras pessoas, explicitando algumas vulnerabilidades às quais está sujeito. Destaca-se, com isso, que o conceito de vulnerabilidade auxilia na análise dessas inter-relações nas dimensões individuais, coletivas e conjuntural-institucionais ${ }^{13}$.

\section{Refletir sobre o lugar de fala e as crises de interpretação}

A empatia consiste em perceber afetos e compartilhálos com a outra pessoa. Entretanto, observa-se que os(as) profissionais tendem a reproduzir, na prática de ensino e cuidado, uma série de violências e preconceitos presentes na sociedade ${ }^{5}$. No caso de Jonas, o cuidado centrado na pessoa e de forma afirmativa não foi experienciado, reproduzindo práticas consideras como "crise de interpretações". Segundo Valla $^{14}$, essa crise ocorre quando se utiliza de experiências e vivências pessoais, em geral advindas de lugares sociais de privilégio, para codificar as necessidades dos outros. Esse processo tende a não ser bem-sucedido quando a perspectiva de cuidado perpassa paradigmas hierárquicos de raça, gênero ou sexualidade. Ou seja, um paradigma que valoriza a raça branca, o gênero cis e masculino e a heterossexualidade.

Cuidar de Jonas, portanto, envolve reconhecer que as vivências e os lugares de fala de pessoas brancas e negras, heterossexuais e LGBTI+ são, infelizmente ainda, diferentes em nossa sociedade, sendo alguns detentores de privilégios, o que provoca crises de interpretação. Proporcionar ao(à) estudante refletir sobre seu lugar de fala ${ }^{15}$ nos processos de cuidado torna possível problematizar as responsabilidades e possibilidades de construir alianças terapêuticas que sejam mais libertárias e menos estigmatizantes.

Outra ação estratégica para a promoção de um cuidado centrado na pessoa e de forma afirmativa para pessoas LGBTI+ é construir ambientes acadêmicos que acolham a diversidade e promovam vivências com o(a)(s) diferente(s). Um exemplo são as Políticas Afirmativas que têm ampliado o acesso ao ensino superior de populações marginalizadas, como negros e transexuais.

\section{Realizar o raciocínio clínico-epidemiológico considerando o contexto LGBTI+}

Um projeto terapêutico necessita de definição conjunta do profissional de saúde com o(a) usuário(a) sobre as questões a serem abordadas para a promoção, prevenção e recuperação da saúde. Se esse processo ocorre de maneira unilateral pelo profissional de saúde, existe um risco de questões importantes não serem percebidas ou consideradas, como na situação descrita. Quando Jonas, homem negro gay, apresenta quadro clínico compatível com ansiedade, é necessário saber que esse grupo está mais exposto a situações que aumentam as chances de suicídio e de ser vítima pela violência, como abuso sexual por parte do parceiro, menor rede de apoio social e familiar, maior dificuldade de expor sua situação de violência por ser um relacionamento homoafetivo e insegurança econômica ${ }^{2,16}$.

\section{"SER OU NÃO SER, EIS A QUESTÃO(?)": AS PRESSUPOSIÇÕES E OS CUIDADOS}

-Cena 2: Lorena (mulher trans parda) chegaà emergência, acompanhada da esposa Rafaela (mulher cis branca, gestante de 28 semanas), queixando-se de febre, cefaleia e dificuldade respiratória há alguns dias. Após avaliação médica, Lorena precisa ser internada. Ao verificar que seu nome de registro é Luís, a equipe passa a chamá-la pelo nome de registro e solicita vaga na ala masculina. Ao comunicar a situação da paciente a Rafaela, o médico informa que realizará um teste de HIV, uma vez que se trata de uma mulher trans. Rafaela questiona a realização do exame e queixa-se de que o médico sequer examinou a esposa. Apesar da argumentação de Rafaela, a equipe insiste e realiza a sorologia sem o consentimento da paciente/usuária, alegando que "ele" é "grupo de risco".

\section{Identificar a LGBTI+fobia como determinante social do} processo saúde-doença

Lorena foi vítima de LGBTI+fobia pelos profissionais de saúde que a atenderam, tanto pelo não reconhecimento do seu nome social quanto pela pressuposição de uma 
infecção sexualmente transmissível (IST) - no caso, o HIV/ Aids. A recorrência desses fatos faz com que esse segmento populacional deixe de procurar os serviços de saúde, só os acessando em situações críticas e ficando desassistido em cuidados básicos ${ }^{17}$.

A LGBTI+fobia é um determinante social do processo saúde-doença e precisa ser abordado ao longo da graduação em Medicina, uma vez que, conforme as DCN, a formação médica deverá ser orientada por meio da concepção da determinação social do processo saúde-doença1. Assim como no racismo, a LGBTI+fobia pressupõe que o outro é destituído de humanidade e não é digno dos mesmos direitos. Essa prática se constitui como um processo estruturante e mantenedor da marginalização e exclusão e ainda culpa as vítimas pela sua condição de vulnerabilidade, invisibilizando a violência estrutural ${ }^{18}$.

Além disso, há a LGBTI+fobia internalizada, um outro tipo de violência, que é o processo pelo qual o indivíduo desvaloriza a si próprio (e os outros) em decorrência de sua orientação sexual ou identidade de gênero não cis-heterossexual. É construída a partir do estigma e da marginalização que se manifesta por negação ou tentativas de mudança da orientação sexual ou identidade, fracasso escolar ou sucesso escolar e/ou profissional excepcional (como forma de compensação) para ser aceito e reconhecido, baixa autoestima, comportamentos de risco, entre outros ${ }^{19}$. Dessa forma, os processos de ensinoaprendizagem ao longo da formação médica devem também estar atentos para essas violências, pois impactarão a saúde e o bem estar do(a) educando(a).

Destaca-se também que, nos processos formativos, a LGBTI+fobia se manifesta quando não se abordam ou se mencionam marginalmente e/ou de forma estigmatizante as questões de gênero e sexualidade, que reiteram a cisheteronorma e invisibilizam essa população. Como resultado, profissionais serão inadequadamente formados(as) para cuidar das pessoas LGBTI+ ${ }^{20,21}$. Outro exemplo disso é o ocultamento da identidade de gênero e/ou orientação sexual no encontro clínico por causa do medo de preconceito e da falta de respeito ${ }^{22,23}$. Para que essas violências não aconteçam, há necessidade também de defender e respeitar a vida e os direitos das pessoas. Assim, o(a) profissional deve ser capaz de acolher e validar o sofrimento das pessoas LGBTI+ e, para além disso, pensar maneiras de garantir diagnósticos e itinerários terapêuticos não agressivos e opressores, o que não ocorreu nas cenas 1 e 2 .

\section{Praticar a humildade cultural}

A relação entre profissionais de saúde e usuários(as) é construída a partir do encontro de sujeitos distintos, que trazem suas bagagens socioculturais próprias. Além de reconhecerem e respeitarem os saberes e as vivências que não Ihes são familiares, os(as) estudantes precisam aprender que cada indivíduo constrói para si um modelo explicativo de seu processo de saúde-adoecimento e que acessá-lo é fundamental para o plano terapêutico. É preciso ser flexível e humilde para reconhecer para si e para o(a) outro(a) o desconhecido e se permitir aprender com o(a) outro(a) e estar disposto a pesquisar informações para melhorar o cuidado e a prática profissional ${ }^{24}$. Refletir também sobre processos intencionais ou não de reprodução de racismo, sexismo, classismo e LGBTI+fobia é uma estratégia para promover o cuidado nesses encontros.

Estereótipos construídos a partir da perspectiva e do discurso hegemônico são formas de reprodução de violências, como descrito na cena 1 por meio da pressuposição de "grupos de risco". É essencial propor ações educativas para desconstruir essas imagens. $\mathrm{O}(\mathrm{A})$ estudante deve problematizar as variadas formas de viver e sofrer e compreender que, por vezes, as mesmas dimensões culturais serão experienciadas de formas diferentes por cada pessoa. Ser culturalmente humilde é ser capaz de refletir sobre os próprios preconceitos, acolhêlos, pensar caminhos para desconstruí-los e entender que a promoção do cuidado integral é um contínuo processo de trocas e aprendizagens, o que reitera a importância do "aprender a aprender", descrito nas DCN".

\section{Promover a saúde reprodutiva e sexual LGBTI+}

Pessoas LGBTI+ podem desejar filhos(as) e podem engravidar de forma indesejada. Porisso, o(a)estudante precisa aprender sobre cuidados reprodutivos para pessoas trans, por exemplo, que desejam ter filhos(as), afinal a hormonização pode reduzir a fertilidade. Também devem saber orientar métodos contraceptivos para pessoas trans, pois hormônios para transformações corporais não são anticoncepcionais. Ademais, a educação sobre saúde reprodutiva e sexual LGBTI+ promove a redução da vulnerabilidade de mulheres bissexuais, que têm altos índices de gravidez indesejada e de uso de anticoncepção de emergência ${ }^{23}$. Cuidados específicos para prevenção de IST, como métodos para o sexo vulva-vulva e sexo anal, gestão do risco para prevenção do HIV e combate à sorofobia (aversão e culpabilização de pessoas vivendo com HIV), são assuntos que devem fazer parte das atividades curriculares sobre saúde sexual ${ }^{25}$.

Na situação descrita, ademais, a gestação de Rafaela, uma mulher cis lésbica, não é acolhida ou legitimada pela equipe de saúde. Seu cuidado em saúde, assim como o de Lorena, é atravessado pela LGBTI+fobia em intersecção com o sexismo, que permeia a experiência social de mulheres 
não heterossexuais. Quando se abordam direitos sexuais e reprodutivos de mulheres no Brasil, inclusive dentro do grupo LGBTI+, é essencial considerar a opressão imposta pelo sistema patriarcal que condiciona mulheres a um lócus de subalternidade e subjugação. Incluir no processo de ensinoaprendizagem a perspectiva das iniquidades de gênero e da sobrecarga imposta às mulheres como fruto do sexismo é um caminho para a produção de um cuidado em saúde atento a tais desigualdades e ativo na construção de pontes para a equidade de gênero ${ }^{10,12}$.

\section{“SOMOS TODOS IGUAIS(?)": O CONTEXTO, O ENSINO E O CUIDADO}

- Cena 3: Reunião de equipe da unidade básica de saúde. A gerente da unidade relata que a Secretaria de Saúde solicitou mapeamento telefônico de idosos(as) que, neste momento de pandemia, estão sozinhos(as) em casa. Após levantamento inicial, o caso do Pedro, homem cis branco de 70 anos, recém-viúvo do segundo casamento - o esposo faleceu no início de 2020 -, é debatido na reunião de equipe, pois há alguns anos não tem contato com a família, a ex-esposa e os filhos do primeiro casamento. Pedro relatou, durante a ligação telefônica, o medo de ir para uma instituição de longa permanência para idosos(as) e ter que voltar para o "armário". Quando a bissexualidade é abordada na reunião, você se questiona sobre o que aprendeu sobre isso em sua formação, recordando-se que ouvia com frequência que "bissexual é o gay enrustido". Quando a equipe foi questionada sobre o que poderia ser feito em relação à orientação sexual, a maioria reitera o discurso de que "todos são iguais e que nada deveria ser feito de especial".

\section{Cuidar das famílias LGBTI+}

As $\mathrm{DCN}_{3}$ no artigo $5^{\circ}$ mencionam que a integralidade e a humanização do cuidado devem considerar os projetos terapêuticos compartilhados, garantindo a autonomia das pessoas, das famílias e dos casais LGBTI+. Estes devem fazer parte do ensino da abordagem familiar, não como um tipo alternativo ou marginal aos arranjos cis-heterossexuais, mas com a mesma importância. Afinal, será que não existem pessoas LGBTI+ nas famílias ou elas estão apenas escondidas? Estudos demonstram que em torno de $2 \%-6 \%$ da população é LGBTI+, possivelmente com dados subestimados ${ }^{26}$.

Muitos ao saírem do "armário" 27 foram expulsos de casa ou têm pouca intimidade com a família consanguínea, o que os leva a optar pela constituição de "famílias de escolha". Geralmente são amigos(as) com vínculos afetivos que se apoiam. É importante que o(a) estudante aprenda a incluílos(as) nos cuidados dos(as) pacientes LGBTI+, como expresso no eixo de "Atenção em Saúde" e "Gestão em Saúde" das $\mathrm{DCN}^{3}$. Na cena 3, o abandono familiar decorrente do fato de o sujeito ser LGBTI+ é um agravante do isolamento social. Além disso, o próprio envelhecimento LGBTI+ e o luto ${ }^{28}$ pela perda da parceria corroboram a fragilidade na rede de apoio. Esses são temas pouco abordados e pesquisados e que poderiam fazer parte também de projetos de extensão e iniciação científica, conforme o eixo de "Educação em Saúde" das DCN³.

\section{Apoiar as fases do ciclo de vida LGBTI+ e a saída do "armário"}

Paulino, Rasera et al. ${ }^{23}$ problematizam o discurso da "não diferença” no cuidado em saúde da população LGBTI+. O processo de silenciamento das demandas específicas em saúde, como pelo discurso de que "todos(as) são iguais", reitera as iniquidades e mantém determinados indivíduos e coletivos em desvantagens. Como educandos(as), educadores(as) e promotores(as) do cuidado em saúde, é preciso reiterar que todo(a) e qualquer cidadão(ã) tem "o direito de ser diferente quando a igualdade nos descaracteriza e o direito de ser igual quando a diferença nos inferioriza" (p. 21) ${ }^{29}$.

A cena 3 apresenta uma violência estrutural que é expressa, por exemplo, nas "piadas" LGBTI+fóbicas. Dizer que o bissexual é um gay "enrustido" insere a bissexualidade no campo da inexistência e da inteligibilidade, e reproduz também a ideia de uma homossexualidade "escondida", que não pode ser revelada nem vivida. A experiência no "armário" 27 ao longo dos processos formativos explicita o "pano de fundo" da formação em saúde que (re)produz e naturaliza a cis-heterossexualidade presumida e compulsória5. Problematizar essas "piadas" e situações de violência estrutural potencializa a compreensão dos corpos (des)controlados na formação e no cuidado em saúde, bem como as estratégias para uma formação humanista, crítica, reflexiva e com responsabilidade social³.

\section{Trabalhar em equipe e gerir o cuidado individual e da comunidade}

As três situações relatadas exemplificam a importância de pensar em ações multiprofissionais para um cuidado que promova os princípios e as diretrizes do Sistema Único de Saúde (SUS). O Projeto Terapêutico Singular é uma ferramenta para o trabalho em equipe, assim como o Genograma e o Ecomapa $^{30}$, que podem auxiliar na compreensão da rede de apoio de Pedro, por exemplo, apontando o isolamento social relacionado à não aceitação familiar e ao medo de ir para uma instituição de longa permanência para idosos(as).

O diagnóstico comunitário, por meio de escuta ativa e estimativas rápidas, é imprescindível para garantir o olhar ampliado sobre as necessidades de saúde ${ }^{30}$. $\mathrm{O}(\mathrm{A})$ estudante 
deve saber acessar fontes de informações, como indicadores e dados demográficos relacionados à orientação sexual e à identidade de gênero, para ampliar esse diagnóstico comunitário. Um exemplo é a afirmativa/o "diagnóstico" de que mulheres cis lésbicas não acessam a unidade de saúde, pois não se sentem acolhidas. Para isso, o(a) estudante precisa conhecer: a proporção desse grupo que frequenta a unidade; como o território lida com as sexualidades não cis-heteronormativas; e como são as experiências dessas mulheres no serviço de saúde e seus itinerários terapêuticos.

Muitas vezes o plano de cuidados envolve apenas criar espaços de troca, de escuta e de acolhimento entre pessoas que vivem dinâmicas sociais parecidas, mas que não se sentem à vontade para falar sobre essas opressões em qualquer espaço não protegido. Promover ou tornar possível, por exemplo, grupos de mulheres negras, de vítimas de violência e de pessoas transexuais em processo de transição pode ser potente para estimular redes de apoio, desconstruir isolamentos e promover autonomia e autocuidado. É necessário que o(a) futuro(a) profissional se perceba como um instrumento de transformação social dentro da instituição de ensino e saúde.

\section{CONSIDERAÇÕES FINAIS}

A pandemia da Covid-19 explicitou a necessidade de considerarmos um ensino e cuidado em saúde que promovam a diversidade de grupos sociais historicamente marginalizados, como das pessoas LGBTI+. Este ensaio exemplifica como é possível abordar a saúde LGBTI+ a partir de situações vivenciadas durante a pandemia da Covid-19. Entretanto, é necessário ter clareza de como as DCN se traduzem em competências específicas sobre saúde LGBTI+. Algumas competências podem ser trabalhadas nesse contexto, como: visibilizar a pessoa LGBTI+ e praticar a semiologia afirmativa, conhecer conceitos de interseccionalidades e vulnerabilidades LGBTI+, refletir sobre o lugar de fala e as crises de interpretação, realizar o raciocínio clínico-epidemiológico considerando o contexto LGBTI+, identificar a LGBTI+fobia como determinante social do processo saúde-doença, prevenir e combater a LGBTI+fobia nas escolas médicas e nos serviços de saúde, promover a defesa da vida e dos direitos das pessoas, praticar a humildade cultural, cuidar das famílias LGBTI+, trabalhar em equipe e gerir o cuidado individual e da comunidade. Trata-se de estratégias que podem tornar os currículos mais acolhedores e compromissados com as necessidades de saúde dessa população.

\section{AGRADECIMENTOS}

Agradecemos a todos(as) os(as) integrantes dos grupos de trabalho Populações (In)Visibilizadas e Diversidades da Associação Brasileira de Educação Médica (Abem) e os Grupos de Trabalho Saúde da População Negra e Gênero, Sexualidade, Diversidade e Direitos da Sociedade Brasileira de Medicina de Família e Comunidade (SBMFC). Agradecemos também a todas as pessoas que de alguma forma apoiam essa luta.

\section{CONTRIBUIÇÃO DOS AUTORES}

Todos(as) os(as) autores(as) contribuíram em todas as etapas de redação e revisão do ensaio.

\section{CONFLITO DE INTERESSES}

Os(as) autores(as) declaram não haver conflito de interesses neste ensaio.

\section{REFERÊNCIAS}

1. Brasil. Resolução $\mathrm{n}^{\circ}$ 3, de 20 de junho de 2014. Institui Diretrizes Curriculares Nacionais do Curso de Graduação em Medicina e dá outras providências. Diário Oficial da União, Brasília; 23 de junho de 2014; Seção 1 , p. 8-11.

2. United Nations Human Rights, Office of the Hight Commissioner. COVID-19 and the human rights of LGBTI people. 2020 [acesso em 10 ago 2020]. Disponível em: https://www.ohchr.org/Documents/Issues/LGBT/ LGBTIpeople.pdf.

3. Baker K, Beagan B. Making assumptions, making space: an anthropological critique of cultural competency and its relevance to queer patients. Med Anthropol Q. 2014;28(4):578-98.

4. Moscheta MD, Fébole DS, Anzolin B. Visibilidade seletiva: a influência da heterossexualidade compulsória nos cuidados em saúde de homens gays e mulheres lésbicas e bissexuais. Saúde Transform Soc. 2016;7(3):71-83.

5. Raimondi GA, Teixeira FD, Moreira C, Barros NF. Corpos (não) controlados: efeitos dos discursos sobre sexualidades em uma escola médica brasileira. Rev Bras Educ Med. 2019;43(3):16-26.

6. Wenceslau LD, Fonseca VK da, Dutra LA, Caldeira LG. Um roteiro de entrevista clínica centrada na pessoa para a graduação médica. Rev Bras Med Fam Comunidade. 2020;15(42):2154.

7. Nogueira O. Preconceito racial de marca e preconceito racial de origem - sugestão de um quadro de referência para interpretação do material sobre relações raciais no Brasil. Tempo social - revista de sociologia da USP. 2006;19(1):287-308.

8. Instituto Brasileiro de Geografia e Estatística. Desigualdades sociais por cor ou raça no Brasil: notas técnicas [Estudos e Pesquisas, Informação Demográfica e Socioeconômica, no 41]. Rio de Janeiro: IBGE; 2019.

9. Akotirene C. Interseccionalidade. São Paulo: Pólen; 2018.

10. Brasil. Política Nacional de Atenção Integral à Saúde da Mulher: princípios e diretrizes. Brasília: Ministério da Saúde; 2004.

11. Brasil. Política Nacional de Atenção Integral à Saúde da População Negra: uma política do SUS. Brasília: Ministério da Saúde; 2013.

12. Brasil. Política Nacional de Saúde Integral de Lésbicas, Gays, Bissexuais, Travestis e Transexuais. Brasília: Ministério da Saúde; 2013.

13. Ayres JR, França Júnior I, Calazans GJ, Saletti Filho HC. O conceito de vulnerabilidade e as práticas de saúde: novas perspectivas e desafios. Promoção da Saúde: Conceitos, Reflexões, Tendências. 2003;2:121-44.

14. Valla VV. A crise de interpretação é nossa: procurando compreender a fala das classes subalternas. Educação \& Realidade. 1996;21(2):177-90.

15. Ribeiro D. O que é lugar de fala? Belo Horizonte: Letramento; 2017.

16. Brasil. Óbitos por suicídio entre adolescentes e jovens negros: 2012 a 2016. Brasília: Universidade de Brasília, Ministério da Saúde; 2018.

17. Silva LK, Silva AL, Coelho AA, Martiniano CS. Uso do nome social no Sistema Único de Saúde: elementos para o debate sobre a assistência prestada a travestis e transexuais. Physis. 2017;27:835-46. 
18. Borrillo D. História e crítica de um preconceito. São Paulo: Autêntica; 2010.

19. Antunes PP. Homofobia internalizada: o preconceito do homossexual contra si mesmo. São Paulo: Annablume; 2017.

20. Rufino AC, Madeiro AP. Ensino da sexualidade na formação médica no Brasil. Einstein (São Paulo). 2015;13(1):vii-i.

21. Raimondi GA, Abreu YR, Borges IM, Silva GB, Hattori WT, Paulino DB. Gênero e sexualidade nas escolas médicas federais do Brasil: uma análise de projetos pedagógicos curriculares. Rev Bras Educ Med. 2020;44(2):e045.

22. Gomes R, Murta D, Facchini R, Meneghel SN. Gênero, direitos sexuais e suas implicações na saúde. Cien Saude Colet. 2018;23:1997-2006.

23. Paulino DB, Rasera EF, Teixeira FD. Discursos sobre o cuidado em saúde de Lésbicas, Gays, Bissexuais, Travestis, Transexuais (LGBT) entre médicas(os) da Estratégia Saúde da Família. Interface Comun Saúde Educ. 2019;23:e180279.

24. Tervalon M, Murray-Garcia J. Cultural humility versus cultural competence: a critical distinction in defining physician training outcomes in multicultural education. J Health Care Poor Underserved. 1998;9(2):117-25.
25. Lopes Junior A, Amorim APA, Ferron MM. Sexualidade e diversidade. In: Gusso G, Lopes JMC, Dias LC. Tratado de medicina de família e comunidade. Princípios, formação e prática. za ed. Porto Alegre: Artmed; 2019. p. 663-74.

26. Bradford J, Mayer KH. What we know and don't know about LGBT demographics: informing clinical practice. In: Makadon HJ, Mayer K. The Fenway guide to lesbian, gay, bisexual, and transgender health. American College of Physicians Press: Philadelphia; 2008. p 28.

27. Sedgwick EK. A epistemologia do armário. Cadernos pagu. 2007;(28):19-54.

28. Henning CE. Gerontologia LGBT: velhice, gênero, sexualidade e a constituição dos “idosos LGBT”. Horizontes Antropológicos. 2017;31(47):283-323.

29. Mantoan MTE. Inclusão escolar: o que é? por quê? como fazer? São Paulo: Moderna, 2006.

30. Santos KK, de Figueiredo CR, de Paiva KM, Campolina LR, Barbosa AA, Santos AS. Ferramentas de abordagem familiar: uma experiência do cuidado multiprofissional no âmbito da estratégia saúde da família. Revista da Universidade Vale do Rio Verde. 2015;13(2):377-87. 